\title{
EVALUATION OF MOVING OBJECT DETECTION BASED ON VARIOUS INPUT NOISE USING FIXED CAMERA
}

\author{
N.Kiaee ${ }^{\text {a }}$, E. Hashemizadeh ${ }^{\text {b, } * \text { N.Zarrinpanjeh }}{ }^{\mathrm{c}}$ \\ a Department of Computer Enginnering, KarajBranch, Islamic Azad University, Karaj, Iran - nadiakiaee @yahoo.com \\ b Department of Mathematics, Karaj Branch, Islamic Azad University, Karaj, Iran - hashemizadeh@kiau.ac.ir \\ c Department of Civil Engineering, Qazvin Branch, Islamic Azad University, Qazvin, Iran - nzarrin@qiau.ac.ir
}

KEY WORDS: Background subtraction, Morphological operation, Moving object detection, Computer vision, Video surveillance, Fixed camera

\begin{abstract}
:
Detecting and tracking objects in video has been as a research area of interest in the field of image processing and computer vision. This paper evaluates the performance of a novel method for object detection algorithm in video sequences. This process helps us to know the advantage of this method which is being used. The proposed framework compares the correct and wrong detection percentage of this algorithm. This method was evaluated with the collected data in the field of urban transport which include car and pedestrian in fixed camera situation. The results show that the accuracy of the algorithm will decreases because of image resolution reduction.
\end{abstract}

\section{INTRODUCTION}

Object detection is an important task within the field of computer vision due video surveillance, traffic monitoring, vehicle navigation, robotics, 3D reconstruction and content based indexing and retrieval. Each visual system, which is involved with tracking, detection of moving objects and interpretation in images, needs to segment the corresponding areas of moving object see (Wang, Yuan et al. 2015) and (KaewTrakulPong and Bowden 2003).

A popular method for detection of moving objects is background subtraction. Background subtraction is an essential step in many machine vision systems. In this way, detection of moving objects without any prior knowledge of the objects is applied. Background subtraction has been studied widely since 1990. This method detects the moving areas in an image with differences of the current frame and the pixels of the background model. While the background is static, this method is easy to implement and has the reasonable output results (Sobral and Vacavant 2014) and (Akula, Khanna et al. 2014). Many algorithms have designed to segment foreground objects from the background sequence. Mahesh et al in (Pawaskar, Narkhede et al. 2014) proposed a "fast and reliable algorithm for moving object detection". It provides the implementation of an efficient object detection algorithm that can be employed in real time embedded systems due to its fast processing. First it will detect moving object based on background subtraction. Then Morphological operation is used for further processing to remove noise and to preserve the shape of moving object. In proposed algorithm complexity of calculation is avoided. Convolution operation is applied to the binary image so that parallel architecture of embedded system will produce result very fast. Binary image is convolved with binary numbered mask, causes limited memory requirement which is allowing the implementation of this system over low-cost FPGAs. Darshan et al presented an algorithm for Detection and Tracking of Multiple Moving Objects in Real Time in (Barcellos, Bouvié et al. 2015). This method is robust in various environments including indoor and outdoor scenes and different types of background scenes, because it uses edge-based features and clustering is used which make it insensitive to illumination changes. It is also fast, because the area to be covered in edgebased features is less than region based features and is not much computationally expensive. This system was extensively tested to operate in complex, real world, non-plain and changing background. To evaluate the performance of the proposed tracking system, the pixel distance from centroid of the tracking window is compared with ground truth and has high accuracy. Barcellos et al provided a novel method for detecting and counting vehicles in urban video sequence. They use particle filtering to measure the particle motion coherence. Moving objects are considered as group of sampling particles which have convex shape. After the detection process, the vehicles are counted by using the same colours of them in each adjacent frame. The experimental results of this method with a total of 80000 video frames showed that it can be more reliable (KR and Nasreen).

Closed circuit television (CCTV) commonly is used in surveillance systems and monitoring. CCTV refers to all cameras that are fixed in place and the images are sent to one or more locations. Most of these cameras are used in the protection, surveillance and security monitoring systems. Fixed cameras are the most recognized CCTV solution due to their ease of use and affordability. These cameras are installed to focus on a fixed area with a high resolution live feed. Though these cameras are fixed positioning they are available for installation with a wide range of lenses (Valera and Velastin 2005) and (Davies and Velastin 2005). 
This paper provides an evaluation of object detection based on background subtraction which allows identifying strength and weakness of this method and helps to focus on the remaining problems. However, there is a wide variety of techniques and both the expert and the newcomer to this area can be confused about the benefits and limitations of this method.

The rest of this paper is organized as follows: Section II describes the main process of moving object detection, section III presents evaluation of moving object detection while section IV consists of the conclusion part.

\section{Proposed method for moving object detection}

\subsection{Background modelling using Gaussian mixture model}

In the traffic monitoring systems fried and Russell have model each pixel of the background by using 3 Gaussian mixture corresponding to the road, vehicles and shadows. Gaussian filters are then labelled. The darkest part will be considered as shadow. About the other two parts, the component with the largest variance will be considered as vehicle and the other one as road. These issues have been set for all processes that have been changed too much. In the foreground detection each pixel has been compared with its Gaussian and has been classified correspond to the corresponding Gaussian. This idea is generalized by modelling recent colour characteristics history of per pixel $\left\{\mathrm{X}_{1}, \ldots, \mathrm{X}_{\mathrm{t}}\right\}$ with a mixture of the $\mathrm{k}$ Gaussians. In this method:

First, each pixel in the RGB colour space specified by its intensity. Then the probability of observing the current pixel value is calculated using the following formula:

$P\left(X_{t}\right)=\sum_{i=1}^{K} \omega_{i, t}, \eta\left(X_{t}, \mu_{i, t}, \sum_{i, t}\right)$

In this formula, the parameters are $\mathrm{k}$ is the number of distributions, $\omega_{i, t}$ is the weight associated to the $i^{\text {th }}$ Gaussian at time $t$ with mean $\mu_{i, t}$ and standard deviation $\sum_{i, t}$.

$\eta\left(X_{t}, \mu, \sum_{i, t}\right)=\frac{1}{(2 \pi)^{\frac{\mathrm{n}}{2}}\left|\sum\right|^{\frac{1}{2}}} \mathrm{e}^{-\frac{1}{2}\left(\mathrm{X}_{\mathrm{t}}-\mu\right) \sum^{-1}\left(\mathrm{X}_{\mathrm{t}}-\mu\right)}$

To solve the computational problems, it is assumed that the RGB colour components are independent and have the same variances. Thus, the covariance matrix is in the form of below:

$\sum_{\mathrm{i}, \mathrm{t}}=\sigma_{\mathrm{i}, \mathrm{t}}^{2} \mathrm{I}$

So each pixel is marked by a mixture of $k$ Gaussians. Once the background model is defined. Gaussian mixture parameters must be initialized. The parameters of this model are the number of $K$ Gaussians, the weight $\omega_{i, t}$ which associated to the $i^{\text {th }}$ Gaussian at times $t$, the mean $\mu_{i, t}$ and covariance matrix $\sum_{i, t}$ (Zivkovic 2004) and (Cheng, Yang et al. 2006).

\subsection{Process of moving object detection}

In this paper, an intelligent system for moving object detection is investigated that this process is defined in Figure 1. Generally, video data recorded by a surveillance camera is converted to frames or images. The conventional techniques for extracting moving objects are background subtraction. In order to guarantee the motion detection and achieve better results, we can overcome and reduce the problems of brightness by updating the background model. In this case, the exact background model will be created as shown in Figure 2.

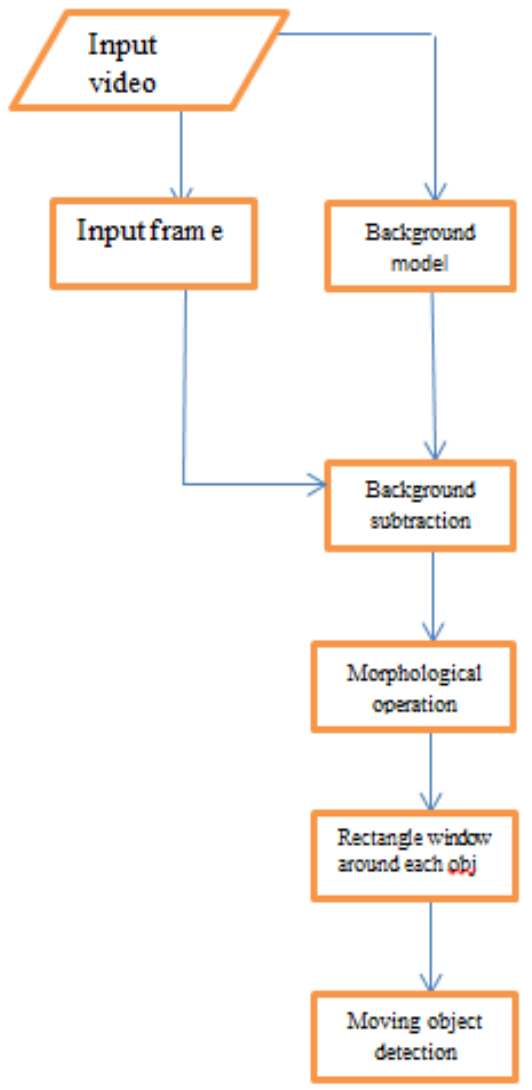

Figure l. Flow chart of moving object detection algorithm

The flowchart of proposed method is presented above. First of all, background model which is considered as the image of road without any moving object from the input video modelled. Then, by subtracting the input frame from the background model, an area of moving object is specified. Morphological operation is used to remove undesirable background noise.

As the main objective of this paper is to detect moving object, we need to determine the moving object area. Although the two dimensional image after morphological operation has no appropriate information, but we use it to find the coordinate of the moving object in the colour frame. So the background and foreground labels refer to the pixels with the value of zero and one respectively. After the labelling step, the maximum and 
minimum coordinate of the rows and columns pixels with one value is applied to colour frames.

In this case, we find the position of moving objects in the colour frame by using the coordinate which obtained from the twodimensional frame. Next, a window rectangle around any detected object is created as shown in Figure 3 (Liang and Juang 2015).

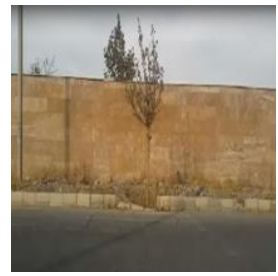

(a)

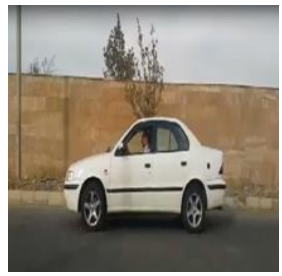

(b)

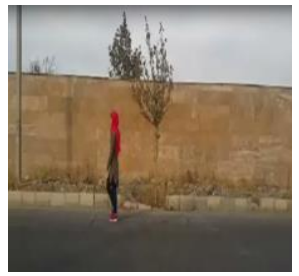

(c)

Figure 2. (a) A model of the estimated background using Gaussian mixture models. (b) a frame which consists of a car (c) a frame which consists of a pedestrian

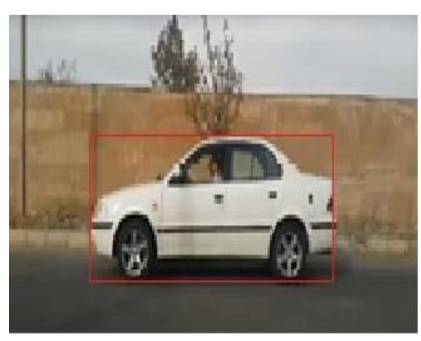

(a)

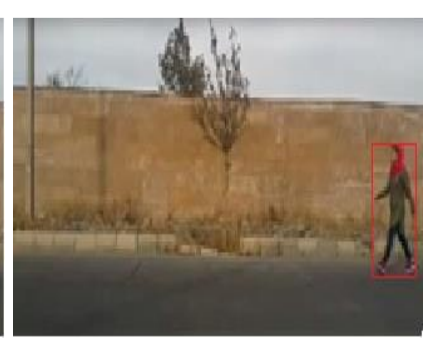

(b)
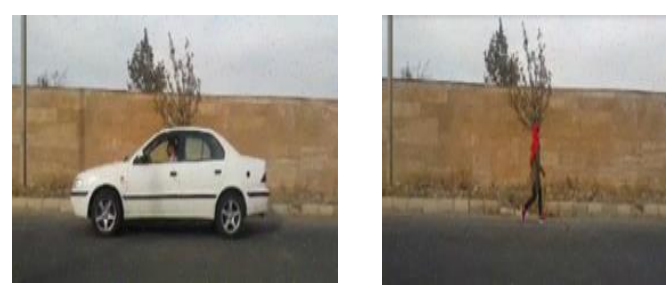

(a)
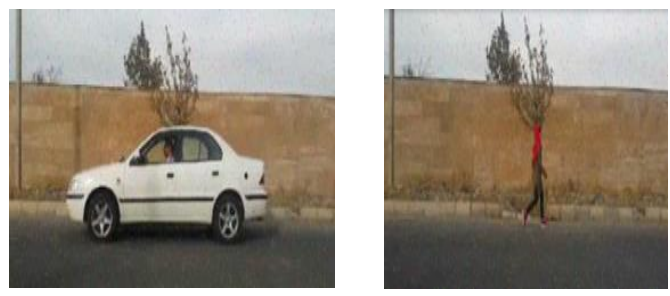

(b)
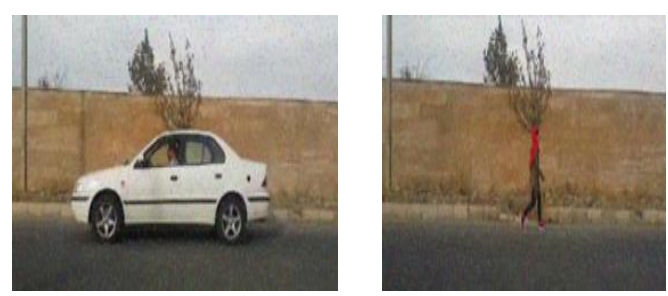

(c)
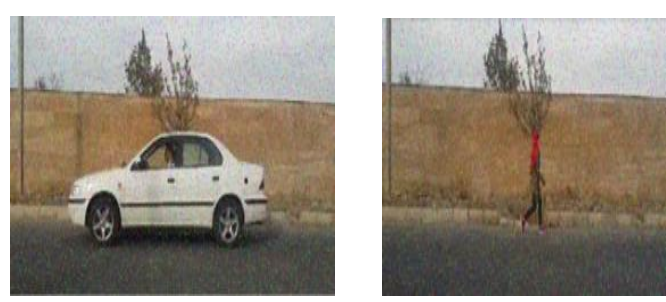

(d)

Figure 4. Resolution reduction effect of various input noise. (a) input noise level=0.01 (b)input noise level=0.02 (c) input noise level=0.04 (d)input noise level $=0.08$

Thus, with different levels of noise, the percentage of successful object detection is investigated in Table 2. According to Figure 4 with increasing the input noise to the system, pedestrian and the success rate of vehicle detection has decreased. In the first step by applying 0 input noises level, the total number of 795 objects are detected and 0 is wrong percentage.

But at the end, with the 0.08 input noises, only 8 objects are detected among the 795 objects and all the other detected objects are cars. Hence it can be concluded, with increasing the input noise, the success detection rate has decreased. Because the pedestrian is more changeable over time rather than the car, the success rate of pedestrian detection is achieved zero. This can be obtained that by increasing the input noise, the vehicle detection based on their size will decrease as shown is Figure 5. 


\begin{tabular}{|c|c|c|}
\hline $\begin{array}{c}\text { Various input } \\
\text { noise }\end{array}$ & $\begin{array}{c}\text { Total number of } \\
\text { detected object }\end{array}$ & Wrong percent \\
\hline 0 & 795 & 0 \\
\hline 0.01 & 583 & $5.31 \%$ \\
\hline 0.02 & 427 & $15.22 \%$ \\
\hline 0.04 & 184 & $45.10 \%$ \\
\hline 0.08 & 41 & $80.48 \%$ \\
\hline
\end{tabular}

Table 2. Result of vehicle detection based on various input noise

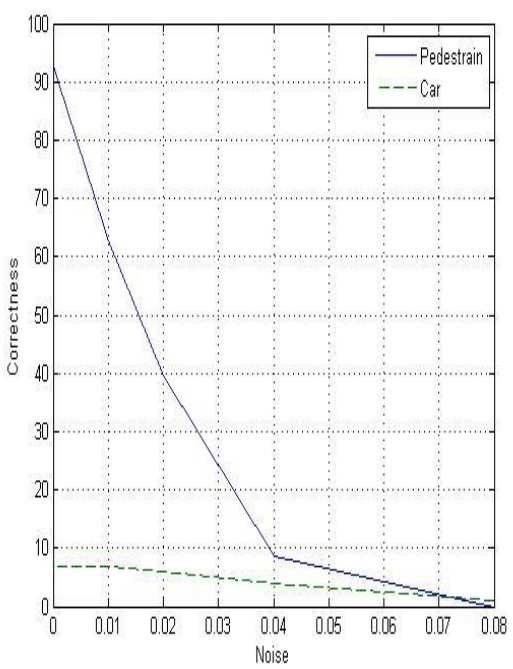

Figure 5. Comparison of various input noise with correct percentage of car and pedestrian detection

In this paper, by limiting the search space to specific location and creating a rectangle around each object, such as car and pedestrian in urban environments, part of the real challenges in computer vision has been explored. The introduced algorithm first detects moving objects automatically and then creates a rectangular area around each object. This method is designed with fixed cameras for intelligent monitoring systems. The results of object detection are more related to the resolution changes of images, especially when the amount of input noise has increased. Finally, it could be concluded that this method is more successful in an ideal day without any undesirable resolution condition.

\section{REFRENCES}

Akula, A., N. Khanna, R. Ghosh, S. Kumar, A. Das and H. Sardana (2014). "Adaptive contour-based statistical background subtraction method for moving target detection in infrared video sequences." Infrared Physics \& Technology 63: 103-109.

Barcellos, P., C. Bouvié, F. L. Escouto and J. Scharcanski (2015). "A novel video based system for detecting and counting vehicles at user-defined virtual loops." Expert Systems with Applications 42(4): 1845-1856.
Cheng, J., J. Yang, Y. Zhou and Y. Cui (2006). "Flexible background mixture models for foreground segmentation." Image and Vision Computing 24(5): 473-482.

Davies, A. C. and S. A. Velastin (2005). "A progress review of intelligent CCTV surveillance systems." Proc. IEEE IDAACS: 417-423.

KaewTrakulPong, P. and R. Bowden (2003). "A real time adaptive visual surveillance system for tracking low-resolution colour targets in dynamically changing scenes." Image and Vision Computing 21(10): 913-929.

KR, D. and A. Nasreen "Detection \& Tracking of Multiple Moving Objects in Real Time."

Liang, C.-W. and C.-F. Juang (2015). "Moving object classification using local shape and HOG features in wavelettransformed space with hierarchical SVM classifiers." Applied Soft Computing 28: 483-497.

Pawaskar, M. M. C., M. N. Narkhede and M. S. S. Athalye (2014). "Detection Of Moving Object Based On Background Subtraction." International Journal of Emerging Trends \& Technology in Computer Science 3(3): 215-218.

Sobral, A. and A. Vacavant (2014). "A comprehensive review of background subtraction algorithms evaluated with synthetic and real videos." Computer Vision and Image Understanding 122: 4-21.

Valera, M. and S. A. Velastin (2005). "Intelligent distributed surveillance systems: a review." IEE Proceedings-Vision, Image and Signal Processing 152(2): 192-204.

Wang, H., C. Yuan and Y. Cai (2015). "Smart road vehicle sensing system based on monocular vision." Optik-International Journal for Light and Electron Optics 126(4): 386-390.

Zivkovic, Z. (2004). Improved adaptive Gaussian mixture model for background subtraction. Pattern Recognition, 2004. ICPR 2004. Proceedings of the 17th International Conference on, IEEE. 